\title{
Human Cerebral Osmolytes during Chronic Hyponatremia A Proton Magnetic Resonance Spectroscopy Study
}

\author{
John S. Videen, ${ }^{\ddagger}$ Thomas Michaelis, ${ }^{\mathbf{5}}$ Patrick Pinto," and Brian D. Ross $\$$ \\ ${ }^{\ddagger}$ Magnetic Resonance Spectroscopy Unit, Huntington Medical Research Institutes, Pasadena, California 91105; ${ }^{8}$ The California Institute \\ of Technology; and "Huntington Memorial Hospital, Pasadena, California 91105
}

\begin{abstract}
The pathogenesis of morbidity associated with hyponatremia is postulated to be determined by the state of intracellular cerebral osmolytes. Previously inaccessible, these metabolites can now be quantitated by proton magnetic resonance spectroscopy. An in vivo quantitative assay of osmolytes was performed in 12 chronic hyponatremic patients (mean serum sodium $120 \mathrm{meq} / \mathrm{liter}$ ) and 10 normal controls. Short echo time proton magnetic resonance spectroscopy of occipital gray and parietal white matter locations revealed dramatic reduction in the concentrations of several metabolites. In gray matter, myo-inositol was most profoundly reduced at $49 \%$ of control value. Choline containing compounds were reduced $36 \%$, creatine/phosphocreatine $19 \%$, and $N$-acetylaspartate $11 \%$ from controls. Similar changes were found in white matter. Recovery of osmolyte concentrations was demonstrated in four patients studied 8-14 wk later. These results are consistent with a reversible osmolyte reduction under hypoosmolar stress in the intact human brain and offer novel suggestions for treatment and monitoring of this common clinical event. (J. Clin. Invest. 1995. 95:788-793.) Key words: hyponatremia • magnetic resonance spectroscopy • osmolytes • brain metabolism - myo-inositol
\end{abstract}

\section{Introduction}

Physicians treating severe hyponatremia have been faced with a dilemma: significant neuropathological disorders and death have been attributed to both hyponatremia and its correction. An abrupt fall in serum sodium concentration $\left(\left[\mathrm{Na}^{+}\right]\right)$is associated with seizures, respiratory arrest, coma, and death (1). Conversely, aggressive treatment of hyponatremia increases the risk for demyelinating lesions of white matter (2-4). Hence, the

A preliminary report of this work was presented at the Second Annual Meeting of the Society of Magnetic Resonance, 1994.

Address correspondence to B. D. Ross, Huntington Medical Research Institutes, 660 South Fair Oaks Avenue, Pasadena, CA 91105. Phone: 818-397-3272; FAX: 818-397-3332. John S. Videen's present address is Division of Nephrology, $9111 \mathrm{H}$ Veterans Affairs Medical Center, 3350 La Jolla Village Drive, San Diego, CA 92161. T. Michaelis' present address is Max-Planck-Institut für biophysikalische Chemie, Postfach 2841, D-3400 Göttingen, Germany.

Received for publication 25 July 1994 and in revised form 22 September 1994.

J. Clin. Invest.

(c) The American Society for Clinical Investigation, Inc.

0021-9738/95/02/0788/06 $\$ 2.00$

Volume 95, February 1995, 788-793 risks of prolonging the hyponatremic state must be balanced against the iatrogenic consequences of prompt correction. Therapeutic recommendations to date have focused on rate and magnitude of correction to match the perceived duration of hyponatremia but frequently lead to a compromise between aggressive and conservative strategies, particularly for a symptomatic patient without an acute history (5-9).

Experimental evidence has linked the development of demyelinating lesions to rapid correction of serum $\left[\mathrm{Na}^{+}\right]$levels after the depletion of intracellular organic osmolytes. Perhaps as an evolutionary adaptation to protect against swelling, the brain differs from other tissues in response to osmotic stress $(10,11)$. Acute hyponatremia is associated with a transient phase of brain swelling followed by brain electrolyte loss to control volume $(10,12)$. Brain water, however, may not approach normal until intracellular organic osmolytes slowly decline over days (13). Systems of organic molecules (e.g., amino acids, polyols, and methylated amines) have evolved to counteract the perturbing effects of solutes on protein structure (14). Rodent models of adapted hyponatremia corrected aggressively with hypertonic saline have demonstrated a lag in the recovery of organic osmolytes and a transient overshoot of brain sodium and chloride (15-17). The elevated ratio of tissue ions relative to organic osmolytes may be the pathogenic mechanism of brain injury (15). Hence, the solution to the clinical dilemma would be to monitor in vivo intracellular osmolytes and correct the $\left[\mathrm{Na}^{+}\right]$ at a rate proportional with their reaccumulation.

Under hyper- and hypotonic conditions, alterations in rat brain concentrations of myo-inositol (mI), ${ }^{1}$ glycerophosphocholine (GPC), creatine/phosphocreatine ( $\mathrm{Cr}$ ), and glutamate/ glutamine (Glx) have identified these compounds as osmolytes $(15,16,18-20)$. Image-guided short echo time proton magnetic resonance spectroscopy (MRS) can resolve these compounds in human brain in vivo $(21,22)$. Alterations of $\mathrm{mI}$ and choline containing compounds ( $\mathrm{Cho}$ ) in the hyperosmolar states of hypernatremia, chronic renal failure, and diabetes mellitus have previously been demonstrated by this laboratory (23-25, unpublished data). These studies applied a novel method of metabolite quantitation and noninvasive assay of cerebral water content. To establish whether these same molecules function as organic osmolytes in the human brain adapting to the hypoosmolar state, we investigated 12 patients with modest hyponatremia. In addition to quantifying the expected changes in cerebral osmolytes, we demonstrate their reversibility. A preliminary account of these studies has been presented (26).

1. Abbreviations used in this paper: $\mathrm{CHF}$, congestive heart failure; Cho, choline containing compounds; $\mathrm{Cr}$, creatine/phosphocreatine; $\mathrm{Glx}$, glycerophosphocholine; mI, myo-inositol; MRS, magnetic resonance spectroscopy; NAA, $N$-acetylaspartate; sI, scyllo-inositol; SIADH, syndrome of inappropriate antidiuretic hormone. 


\section{Methods}

Patients with hyponatremia $\left(\left[\mathrm{Na}^{+}\right]\right.$below $125 \mathrm{meq} /$ liter) were identified from inpatient and outpatient chemistry reports of Huntington Memorial Hospital in Pasadena. Those with focal brain lesions, dementia, hepatic failure, renal failure, or diabetes mellitus were excluded as these are known to alter cerebral $\mathrm{mI}$ and $\mathrm{Cho}(24,25,27,28)$. The duration and clinical course of the hyponatremia were determined by review of patient records and interview; patients with acute illness $(<1 \mathrm{wk})$ were excluded. Hypoosmolality was confirmed by direct measurement or calculation from serum $\left[\mathrm{Na}^{+}\right]$, urea, and glucose. Magnetic resonance spectroscopy (MRS) was performed within $12 \mathrm{~h}$ of a blood draw to confirm hyponatremia and before significant correction. The study was repeated after an interval of $8-14$ wk in 4 of 12 patients. 10 healthy volunteers of similar age served as controls.

Localized quantitative proton MRS of the brain was performed on a 1.5-T scanner (Signa 4.8, General Electric, Waukeshau, WI) using a STEAM sequence (30-ms TE, 3.0-s TR, 13.7-ms TM, and 64 acquisitions) and processing scheme developed in this laboratory $(29,30)$. After axial T1 localizer MR images were obtained, single voxel (11.3 $\mathrm{ml})$ spectra in the midline occipito-parietal gray matter were acquired in all patients and controls. Brain water compartments were then assayed and a reference spectrum of an external standard acquired. This fully quantitative examination was completed in $30 \mathrm{~min}$. Additional examination of parietal white matter $(12.5 \mathrm{ml})$ was performed in three patients. Because relaxation effects may significantly influence metabolite quantitation by MRS, gray matter $\mathrm{T} 1$ and $\mathrm{T} 2$ relaxation times were determined in hyponatremic individuals and normal controls. Additional TRs of 1.5 and $5.0 \mathrm{~s}$ were used to determine metabolite $\mathrm{T} 1$ and six different echo times (TE) of $30,40,60,90,135$, and $270 \mathrm{~ms}$ to determine metabolite $\mathrm{T} 2$.

Spectra were processed with eddy current correction, removal of residual water signal by a low frequency filter, and apodization before Fourier transformation and semiautomated phasing as previously described (21). Scaling of peak height was accomplished by two methods. The height of the $\mathrm{Cr}$ peak was assigned to 1.00 to provide a relative scale. For absolute scaling, the spectra were multiplied by a correction factor that accounted for percent cerebrospinal fluid in the voxel, coil loading, and voxel size. Summed spectra were obtained by vector summation and scalar division to display an average spectrum. Difference spectra were produced by vector subtraction and magnified with scalar multiplication.

Metabolite amounts were calculated both as a ratio relative to $\mathrm{Cr}$ peak intensity and as absolute concentrations expressed in $\mathrm{mmol} / \mathrm{kg}$ wet weight. Metabolite ratios relative to $\mathrm{Cr}$ were determined in an operator-independent manner from peak amplitudes after LorentzGauss transformation (27). The quantitative schema uses peak integrals and includes a correction for brain compartmentation whereby the voxel was divided into brain water (intra- and extracellular), cerebrospinal fluid, and "dry matter" based on water T2 characteristics (29). Adjustments for $\mathrm{T} 1$ and $\mathrm{T} 2$ relaxation were also used, and the combined method is known to yield reproducible results in close agreement with other in vitro methods (30). Results are expressed as means $\pm S D$, and statistical significance was determined using Student's $t$ test.

\section{Results}

Characteristics of the patients are listed in Table I. The age and ranges of the patient and control groups were nearly identical, $61-85$ and $60-84 \mathrm{yr}$, respectively, but the means were slightly different (76 \pm 7 and $70 \pm 8 \mathrm{yr}$ ). Mean serum $\left[\mathrm{Na}^{+}\right]$of the patient group was $120 \mathrm{meq} /$ liter (normal 135-145). All patients were hypoosmolar with a mean osmolality of $248 \mathrm{mosM} / \mathrm{kg}$ (normal 280-290). The syndrome of inappropriate antidiuretic hormone (SIADH) was defined as persistent hypoosmolality with inappropriate urine concentration, euvolemia, and the ab-
Table I. Patient Characteristics and Etiology of Hyponatremia

\begin{tabular}{cccccl}
\hline Patient & Sex & Age & Serum Na $^{+}$ & Osmolality & \multicolumn{1}{c}{ Etiology } \\
\hline & & $y r$ & meqniter & mosM/kg & \\
1 & M & 61 & 120 & 245 & Pituitary tumor \\
2 & M & 85 & 122 & $248^{*}$ & CHF \\
3 & M & 75 & 117 & $238^{*}$ & SIADH \\
4 & F & 77 & 123 & 254 & CHF/thiazide \\
5 & M & 69 & 121 & $249 *$ & Carbamezipine \\
6 & F & 79 & 121 & 257 & CHF \\
7 & M & 84 & 118 & 250 & SIADH \\
8 & F & 77 & 122 & $245 *$ & SIADH \\
9 & F & 82 & 119 & 248 & CHF \\
10 & M & 79 & 119 & 247 & Fluoxetine \\
11 & F & 75 & 118 & 244 & SIADH \\
12 & F & 66 & 118 & 246 & CHF \\
Mean \pm SD & & $76 \pm 7$ & $120 \pm 2$ & $248 \pm 5$ & \\
& & & & & \\
\hline
\end{tabular}

* Osmolality measured directly or calculated as $2 \times\left[\mathrm{Na}^{+}\right]+$glucose + urea.

sence of systemic illness or medications known to cause hyponatremia. Congestive heart failure (CHF) was believed to be etiologic in 5 of 12 patients. In three patients, hyponatremia improved after discontinuation of medications associated with inappropriate antidiuresis. Symptoms of hyponatremia included malaise $(n=8)$, weakness $(n=7)$, and confusion $(n=3)$ lasting for several days or weeks before presentation. Two patients were asymptomatic. All patients were treated with water restriction, two received demeclocycline, one received hypertonic saline, but none had significantly corrected serum $\left[\mathrm{Na}^{+}\right]$ by the time of MRS. No patient developed adverse neurologic symptoms or the osmotic demyelination syndrome.

Fig. 1 shows representative proton magnetic resonance spectra from gray and white matter in a control and patient 11. These spectra are scaled relative to $\mathrm{Cr}$ peak height. Four major peaks are readily apparent and have been assigned to $\mathrm{N}$-acetylaspartate (NAA), $\mathrm{Cr}$, Cho, and $\mathrm{mI}$. Smaller peaks present in the figures include the alpha and beta/gamma resonances of Glx ( $\alpha$-Glx and $\beta, \gamma$-Glx) and scyllo-inositol (sI). A decrease in $\mathrm{mI}$, sI, and Cho can be appreciated in patient spectra compared with the control in both gray and white matter.

Table II shows that significant and sizable abnormalities in the metabolite amplitude ratios of both gray and white matter are present in patients with moderate hyponatremia. In gray matter, significant reductions in $\mathrm{mI}: \mathrm{Cr}(30 \%)$ Cho: $\mathrm{Cr}(15 \%)$ ratios were noted. NAA:Cr and Glx:Cr were not altered significantly. Similar changes of metabolite ratios in white matter were found $(n=3)$. Glx was not significantly reduced in white matter. An increase in the white matter $\alpha-\mathrm{Glx}$ :Cr ratio unaccompanied by a change in $\beta, \gamma-\mathrm{Glx}: \mathrm{Cr}$ ratio may reflect statistical scatter.

To determine whether these changes in metabolite ratios were due to changes in the concentrations of the relevant metabolites in the brain, 11 of 12 patients underwent a quantitative examination. Gray and white matter data listed in Table III reveal dramatic reductions in metabolite concentrations. As well as the expected decline in the concentrations of $\mathrm{mI}([\mathrm{mI}])$ and Cho ([Cho]), these absolute data also indicate significant reductions in the concentrations of NAA and $\mathrm{Cr}$. In gray matter, the summed 


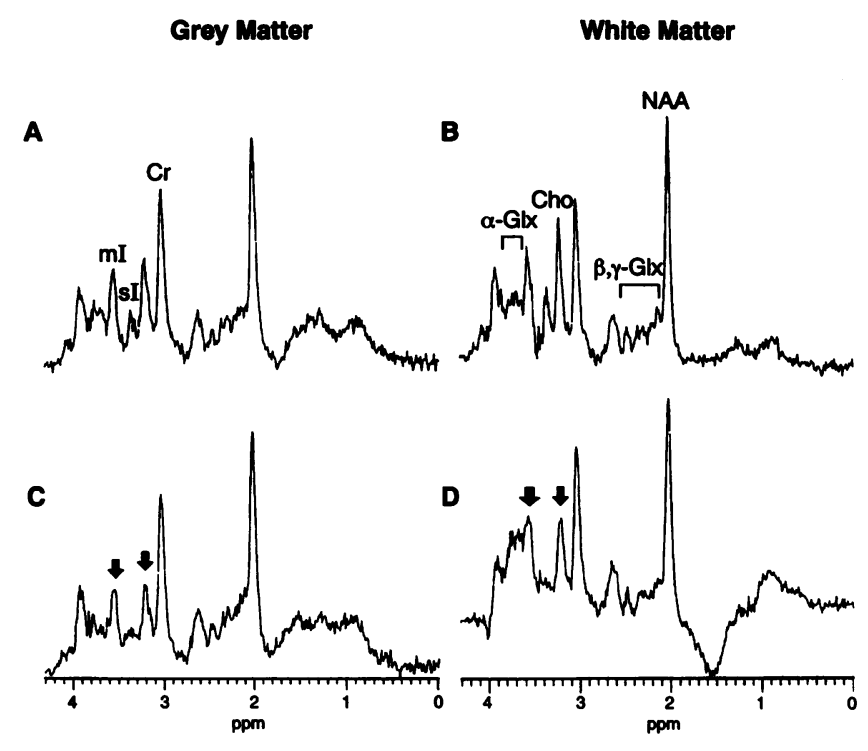

Figure 1. Representative brain proton magnetic resonance spectra from a control and hyponatremic patient. Spectra were scaled to $\mathrm{Cr}$. The top spectra are from gray $(A)$ and white $(B)$ matter regions of a normal control. Peaks representing the four major metabolites are labeled: $N A A$, $\mathrm{N}$-acetylaspartate; $\mathrm{Cr}$, total creatine; $\mathrm{Cho}$, choline containing compounds; $m I$, myo-inositol. Also indicated are the $\alpha$ and $\beta, \gamma$ regions of glutamate plus glutamine $(G l x)$ and an isomer of $\mathrm{mI}$, scyllo-inositol $(s I)$. The lower spectra are those of patient $11,\left[\mathrm{Na}^{+}\right]=118 \mathrm{meq} / \mathrm{liter}$, gray $(C)$ and white matter $(D)$ locations. The decline of $\mathrm{mI}$, Cho, and sI in the hyponatremic patient is evident in both white and gray matter.

change in concentration of these four metabolites is a reduction of $29 \%$, from $28 \pm 4 \mathrm{mmol} / \mathrm{kg}$ in the controls to $20 \pm 3 \mathrm{mmol} / \mathrm{kg}$ in the hyponatremic patients. More than half $(58 \%)$ of this change is due to depletion of $\mathrm{mI}$. Fig. 2 depicts these quantitative changes directly. Here, the summed spectra of patients $(A)$ and controls $(B)$ are displayed with the difference spectrum $(C)$. Note that NAA, Cr, Cho, and $\mathrm{mI}$ all appear as negative peaks as indicated by the quantitative data above. Additionally, a reduction in both the $\alpha$ and $\beta, \gamma$ region of Glx is suggested in the difference spectrum, even though this does not appear as a difference in the metabolite ratios (see Table II).

Four patients were available for follow-up spectroscopic examinations several weeks after the initial study (follow-up $\left[\mathrm{Na}^{+}\right] 131 \pm 6 \mathrm{meq} /$ liter $)$. Only one returned to a normal serum $\left[\mathrm{Na}^{+}\right]$, and this patient's recovery after surgical removal of a pituitary tumor is clearly demonstrated by the initial, follow-

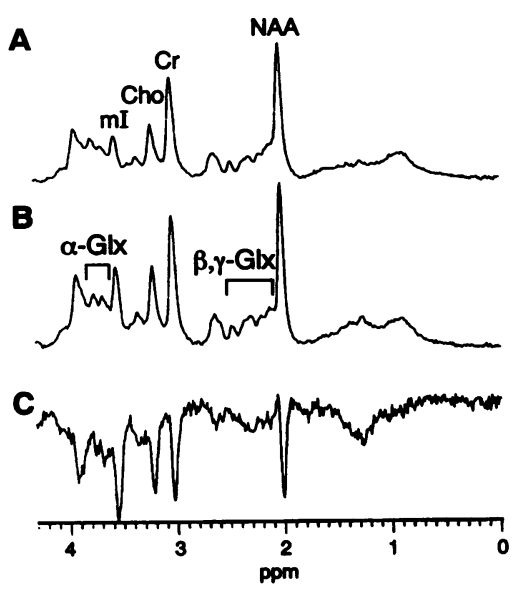

Figure 2. Summed and difference spectra of gray matter in hyponatremia. Individual spectra were processed on an absolute scale (see Methods). Spectra of 11 hyponatremic patients $(A)$ and 10 controls $(B)$ were summed and then subtracted (patients minus controls) to yield the difference spectrum $(C)$, which is enlarged threefold. The negative peaks of the four major metabolites represent a decline in concentration. The

fifth peak at $3.95 \mathrm{ppm}$ represents the methylene protons of creatine and is proportional to the methyl creatine peak at $3.05 \mathrm{ppm}$.

up, and difference spectra depicted in Fig. 3. Other patients with incomplete correction of hyponatremia showed measurable recovery of cerebral osmolytes but retained spectroscopic abnormalities. Fig. 4 summarizes the paired quantitative data of all four patients in terms of percent change from normal metabolite concentrations. Each of the four osmolytes returned toward normal, but with this small number of subjects no statistical correlation between the extent of recovery of serum and cerebral osmolytes could be determined. The smallest extent of recovery was recorded in patient 5 , in whom serum $\mathrm{Na}^{+}$was only marginally corrected. Significant recovery of [NAA], [Cho], and [mI] (but not $[\mathrm{Cr}]$ ) was demonstrable between initial and followup MRS (Table IV). The mean interval between the initial (hyponatremia) and follow-up ("recovery") MRS examinations was $10.8 \mathrm{wk}$, consistent with the expectedly very slow rate of restoration of intracerebral osmotic status.

Spectra obtained from a subset of patients $(n=3)$ at different TR revealed no significant changes in T1 of NAA, Cr, Cho, and $\mathrm{mI}$ from that reported by Kreis et al. (30). Determination of $\mathrm{T} 2$ of NAA, Cr, Cho, and $\mathrm{mI}$ in two patients also did not reveal significant changes, indicating that the changing spectral peak areas determined during hyponatremia reflect changing concentrations. However, due to the severe reduction in [Cho] and $[\mathrm{mI}]$, the determination of their $\mathrm{T} 2$ is less precise.

\section{Discussion}

As far as we are aware, the present results represent the first direct demonstration of quantitative cerebral osmolyte abnor-

Table II. Cerebral Metabolite Ratios in Gray and White Matter of Hyponatremic Patients and Controls

\begin{tabular}{|c|c|c|c|c|c|c|}
\hline & \multicolumn{3}{|c|}{ Gray matter } & \multicolumn{3}{|c|}{ White matter } \\
\hline & Control $(n=10)$ & Hyponatremia $(n=12)$ & $P$ & Control $(n=7)$ & Hyponatremia $(n=3)$ & $P$ \\
\hline NAA:Cr & $1.23 \pm 0.08$ & $1.27 \pm 0.12$ & NS & $1.38 \pm 0.09$ & $1.29 \pm 0.01$ & NS \\
\hline Cho:Cr & $0.60 \pm 0.06$ & $0.51 \pm 0.06$ & $<0.01$ & $0.84 \pm 0.08$ & $0.56 \pm 0.02$ & $<0.001$ \\
\hline $\mathrm{Ml}: \mathrm{Cr}$ & $0.60 \pm 0.07$ & $0.41 \pm 0.05$ & $<0.0001$ & $0.66 \pm 0.04$ & $0.52 \pm 0.04$ & $<0.001$ \\
\hline$\alpha-\mathrm{Glx}: \mathrm{Cr}$ & $0.43 \pm 0.09$ & $0.46 \pm 0.09$ & NS & $0.46 \pm 0.06$ & $0.65 \pm 0.13$ & $<0.01$ \\
\hline$\beta, \gamma-\mathrm{Glx}: \mathrm{Cr}$ & $0.25 \pm 0.04$ & $0.23 \pm 0.06$ & NS & $0.20 \pm 0.03$ & $0.16 \pm 0.06$ & NS \\
\hline
\end{tabular}

Values are means \pm SD. $P$ determined using Student's $t$ test. NS indicates $P>0.05$. 
Table III. Absolute Concentrations of Cerebral Metabolites in Hyponatremic Patients and Controls

\begin{tabular}{|c|c|c|c|c|c|c|}
\hline & \multicolumn{3}{|c|}{ Gray matter } & \multicolumn{3}{|c|}{ White matter } \\
\hline & Control $(n=10)$ & Hyponatremia $(n=11)$ & $P$ & Control $(n=7)$ & Hyponatremia $(n=3)$ & $P$ \\
\hline NAA & $8.84 \pm 1.05$ & $7.87 \pm 1.21$ & $<0.05$ & $8.68 \pm 0.78$ & $7.12 \pm 0.56$ & $<0.01$ \\
\hline $\mathrm{Cr}$ & $8.39 \pm 0.72$ & $6.78 \pm 0.48$ & $<0.0001$ & $6.64 \pm 0.29$ & $5.72 \pm 0.43$ & $<0.01$ \\
\hline Cho & $1.68 \pm 0.30$ & $1.08 \pm 0.12$ & $<0.0001$ & $1.90 \pm 0.20$ & $1.07 \pm 0.15$ & $<0.001$ \\
\hline $\mathrm{mI}$ & $8.97 \pm 1.68$ & $4.58 \pm 1.17$ & $<0.0001$ & $8.04 \pm 0.33$ & $6.42 \pm 0.75$ & $<0.001$ \\
\hline
\end{tabular}

Values are means $\pm \mathrm{SD}$ expressed in $\mathrm{mmol} / \mathrm{kg}$ wet wt. $P$ determined using Student's $t$ test.

malities associated with hyponatremia in humans. Our results are consistent with several earlier animal studies in all of which osmolyte assays were performed in vitro $(15-18,20,31)$. The changes determined by proton MRS are quite sizable and can be readily observed in the individual patient and followed over time.

The details of cerebral osmolyte composition appear similar to those described in the rat brain $(15-17)$. The significant absolute reductions in $\mathrm{mI}(49 \%)$, Cho (36\%), and $\mathrm{Cr}(19 \%)$ in this study are comparable with changes of $\mathrm{mI}, \mathrm{GPC}$, and $\mathrm{Cr}$ in hyponatremic rats $(15,16,18)$. GPC cannot be separately assayed in in vivo proton MRS but comprises up to half of the Cho peak $(32,33)$. Glx is an important osmolyte in rat brain. We are unable to demonstrate a significant reduction in $\mathrm{Glx}: \mathrm{Cr}$ in humans, possibly because of the fall in [ $\mathrm{Cr}$ ]. However, [ Glx ] does appear to be reduced in the difference spectrum (Fig. 2). Although not an osmolyte per se and infrequently measured in studies of osmolar stress, our demonstration of a reduction in [NAA] $(-11 \%)$ is supported in a rat model of acute hyponatremia (31). Alternatively, the NAA peak may be factitiously low secondary to a change of another metabolite underlying the NAA and $\beta, \gamma$-Glx region of the spectrum. Of the four metabolites quantitated in this study by MRS, $\mathrm{mI}$ accounts for $58 \%$ of the total change and is therefore the predominant cerebral osmolyte in the human brain. A minimum estimate of the total cerebral osmolyte change detected by ${ }^{1} \mathrm{H}$ MRS is -6.57 mosM/ $\mathrm{kg}$ in gray matter (see Table III) and $-4.43 \mathrm{mosM} / \mathrm{kg}$ in white matter, representing $16 \%$ and $11 \%$, respectively, of the mean reduction in serum osmolality of $-42 \mathrm{mosM} / \mathrm{kg}$ (Table I). However, the true effect is probably somewhat greater for the following reason. Because [Cr] is significantly reduced, we believe that Glx, the sum of glutamate and glutamine, is similarly reduced by $\sim 20 \%$. Assuming $\mathrm{Glx} \cong 16 \mathrm{mmol} / \mathrm{kg}$, this represents a further reduction of measured cerebral osmolytes of $-3 \mathrm{mosM} / \mathrm{kg}$ and brings the total cerebral osmolyte loss in hyponatremia to $-23.3 \%$ in gray matter and $-18.1 \%$ of the change in plasma osmolality.

Although an increase in brain water that returns toward normal with adaptation to hypoosmolality is expected, there was no observed alteration in the size of the compartments of cerebral water in patients versus controls. Rodent models of chronic hyponatremia have reported a $0.4-6 \%$ increase in brain water content $(10,13,15-17)$. In our patient sample, adaptation may become so complete as to be impossible to detect with present MRS techniques. Alternatively, because the three-compartment model used in our methods is unable to distinguish between intracellular $(\sim 80-90 \%)$ and extracellular $(\sim 10-$ $20 \%$ ) fluid, relative changes in these compartments cannot be excluded. Nevertheless, it is unlikely that the brain water content or ratio of intracellular to extracellular fluid compartments increased enough to completely account for the $20-50 \%$ reduction of osmolytes by dilution; therefore reduced synthesis, increased catabolism, or impaired influx is implied.

There was no correlation between the extent of the reduction in cerebral $[\mathrm{mI}]$ and serum $\left[\mathrm{Na}^{+}\right]$or osmolality $(r<0.3)$. In addition to severity, one would predict duration of hyponatremia to be a significant variable but somewhat difficult to control in a study of this design. Assuming that all of our patients reached a steady state where intracellular osmolytes equilibrated with serum tonicity, the lack of a correlation indicates the presence of other independent factors that contribute to the regulation of the intracellular osmolytes (e.g., malnutrition, glucose metabolism, and presence of other unmeasured osmolytes). It is of particular interest to note that normonatremic patients with hepatic encephalopathy have evidence of altered cerebral osmolyte metabolism by proton MRS (34). Even patients with subclinical hepatic encephalopathy demonstrate reduced $\mathrm{ml}: \mathrm{Cr}$ and $\mathrm{Cho}: \mathrm{Cr}$ but, in contrast with hyponatremics, have elevated $\mathrm{Glx}: \mathrm{Cr}$ (35). Thus the higher observed risk of myelinolysis in alcoholics and cirrhotics may be explained by an independent effect of liver disease on cerebral myo-inositol metabolism $(36,37)$.

Although the time course of recovery of cerebral osmolytes has not been established in the present studies, it is clearly

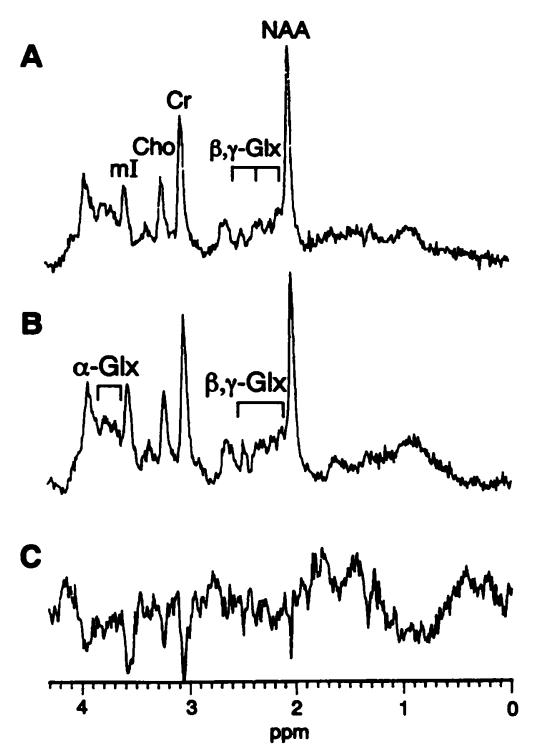

Figure 3. Initial, followup, and difference proton MR spectra in a single patient with hyponatremia. The initial $(A)$ gray matter spectrum was acquired at a $\left[\mathrm{Na}^{+}\right]$ of $120 \mathrm{meq} / \mathrm{liter}$ and the follow-up $(B)$ at 139 meq/liter 14 wk later (patient 1, Table I). The spectra are processed on an absolute scale. The difference spectrum $(C$, initial minus follow-up with $1 \mathrm{~Hz}$ line-broadening) is enlarged threefold. The recovery of $\mathrm{ml}$, $\mathrm{Cho}, \mathrm{Cr}$, and NAA is demonstrated by the corresponding negative peaks in the difference spectrum. 


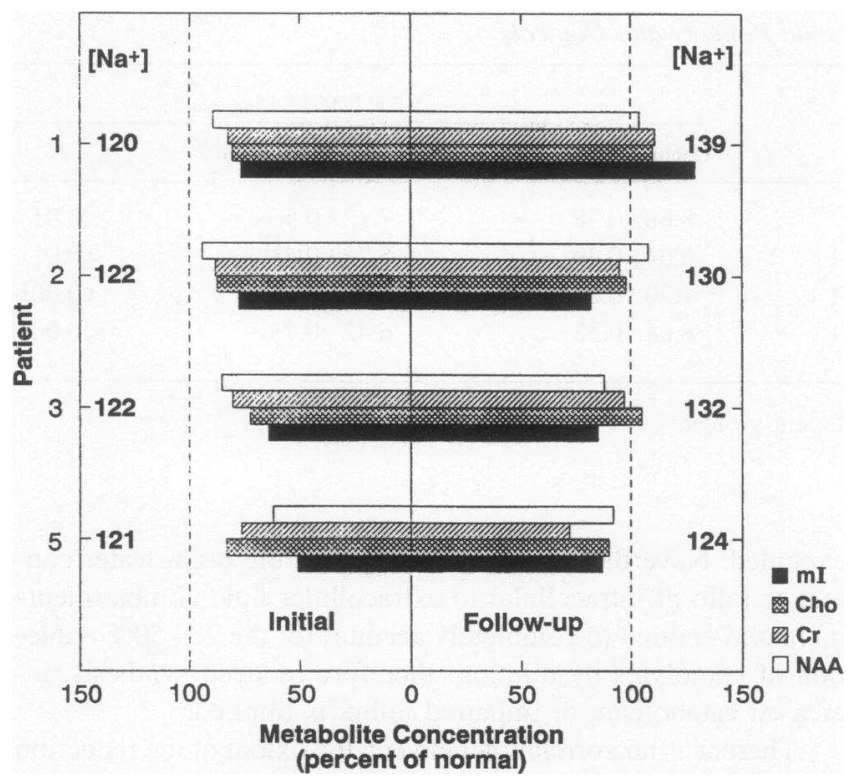

Figure 4. Bar graph of initial and follow-up metabolite concentrations of gray matter in four treated hyponatremic patients. NAA, Cr, Cho, and $\mathrm{MI}$ are quantitated as percentage of the normal metabolite concentration (30). Serum $\left[\mathrm{Na}^{+}\right]$for each study is indicated. The initial osmolyte deficit is indicated by the "gap" between the shaded columns and the $100 \%$ line. Recovery is demonstrated on the righthand side of the figure, as a reduction in this gap. The interval between the initial and followup examinations was 14 wk (patient 1), 11 wk (patient 2), $10 \mathrm{wk}$ (patient 3), and 8 wk (patient 5) (mean $10.8 \mathrm{wk}$ ).

prolonged, being measured in weeks, not days. As pointed out by Narins (38) 8 years ago, the controversy over therapy of symptomatic hyponatremia cannot be solved without a prospective study with stratification of defined groups and careful attention of brain metabolism. It now appears that a tool is available to undertake such a study and guide therapy. One could monitor osmolyte concentrations in symptomatic patients with hyponatremia and treat at a proportional rate. Those patients with unacceptably low osmolytes may require alternative modalities (urea, colchicine, steroids) (39-41) to protect against osmotic stress.

In summary, this study demonstrates the reversible reduction in human cerebral organic osmolyte concentrations in response to hypoosmolar stress predicted by animal studies. MRS of brain metabolism during osmolar stress will ultimately con-

Table IV. Recovery of Cerebral Osmolytes after Treatment of Hyponatremia

\begin{tabular}{lcc}
\hline & Increase & $P$ \\
\hline$\left[\mathrm{Na}^{+}\right]$, meq/liter & $+10 \pm 7$ & $<0.05$ \\
$\mathrm{NAA}, \%$ & $+16 \pm 11$ & $<0.05$ \\
$\mathrm{Cr}, \%$ & $+12 \pm 14$ & $\mathrm{NS}$ \\
$\mathrm{Cho} \%$ & $+20 \pm 13$ & $<0.05$ \\
$\mathrm{mI}, \%$ & $+29 \pm 20$ & $<0.05$
\end{tabular}

Values are means \pm SD. Paired $t$ test $(n=4)$; results of osmolyte assays are expressed as $\%$ of controls. The same convention is applied in Fig. 4. NS $=P>0.05$. tribute to an understanding of basic physiologic and pathologic issues in humans. The changes can be observed in the individual patient with moderate asymptomatic hyponatremia in a noninvasive examination requiring only $30 \mathrm{~min}$ to perform and can therefore be used to monitor clinical states and their response to therapy.

\section{Acknowledgments}

We thank the physicians of Huntington Memorial Hospital for permission to examine patients under their care. Some of the MRS investigations reported in this study were performed by Dr. Rex A. Moats, Truda Shonk, and Dr. Else R. Danielsen. We thank Dr. Patrick Vinay for useful discussion.

This work was supported in part by the National Institutes of Health through a Physician-Scientist Program Award (NIH 2 K12 DK01408) to J. S. Videen under the auspices of the University of California, San Diego. B. D. Ross is grateful to the L. K. Whittier Foundation and the Jameson Foundation for support of the Clinical Magnetic Resonance Unit at Huntington Medical Research Institutes. B. D. Ross is Visiting Associate and T. Michaelis is the James Boswell Research Fellow at the California Institute of Technology.

\section{References}

1. Arieff, A. I. 1986. Hyponatremia, convulsions, respiratory arrest and permanent brain damage after elective surgery in healthy women. N. Engl. J. Med. 314:1529-1535.

2. Sterns, R. H., J. E. Riggs, and S. S. Schochet. 1986. Osmotic demyelination syndrome following correction of hyponatremia. N. Engl. J. Med. 314:15351542.

3. Messert, B., W. W. Orrison, M. J. Hawkins, and C. E. Quagliere. 1979. Central pontine myelinolysis, considerations on etiology, diagnosis and treatment. Neurology. 29:147-160.

4. Burcar, P. J., M. D. Norenberg, and P. R. Yarnell. 1977. Hyponatremia and central pontine myelinolysis. Neurology. 27:223-226.

5. Ayus, J. C., R. K. Kroghapalli, and A. I. Arieff. 1987. Treatment of symptomatic hyponatremia and its relation to brain damage, a prospective study. $N$. Engl. J. Med. 27:1190-1195.

6. Sterns, R. H. 1987. Severe symptomatic hyponatremia: treatment and outcome. Ann. Intern. Med. 107:656-662.

7. Norenberg, M. D. 1981. Association between rise in serum sodium and central pontine myelinolysis. Ann. Neurol. 11:128-135.

8. Berl, T. 1990. Treating hyponatremia: what is all the controversy about? Ann. Intern. Med. 113:417-419.

9. Soupart, A., R. Penninckx, A. Steniut, O. Perier, and G. Decaux. 1992 Treatment of chronic hyponatremia in rats by intravenous saline: comparison of rate versus magnitude of correction. Kidney Int. 42:1662-1667.

10. Rymer, M. M., and R. A. Fishman. 1973. Protective adaptation of brain to water intoxication. Arch. Neurol. 28:49-54.

11. Dila, C. J., and H. M. Pappius. 1972. Cerebral water and electrolytes: an experimental model of inappropriate secretion of antidiuretic hormone. Arch. Neurol. 26:85-90.

12. Melton, J. E., and E. E. Nattie. 1983. Brain and CSF water and ions during dilutional and isosmotic hyponatremia in the rat. Am. J. Physiol. 244:R724-R732.

13. Sterns, R. H., D. J. Thomas, and R. M. Herndon. 1989. Brain dehydration and neurologic deterioration after rapid correction of hyponatremia. Kidney Int. 35:69-75.

14. Yancey, P. H., M. E. Clark, S. C. Hand, R. D. Bowlus, and G. N. Somero. 1982. Living with water stress: evolution of osmolyte systems. Science (Wash. DC). 217:1214-1222.

15. Lien, Y.-H. H., J. I. Shapiro, and L. Chan. 1991. Study of brain electrolytes and organic osmolytes during correction of chronic hyponatremia. J. Clin. Invest. 88:303-309.

16. Verbalis, J. G., and S. R. Gullans. 1993. Rapid correction of hyponatremia produces differential effects on brain osmolyte and electrolyte reaccumulation in rats. Brain Res. 606:19-27.

17. Thurston, J. H., R. E. Hauhart, and J. S. Nelson. 1987. Adaptive decreases in amino acids (taurine in particular), creatine, and electrolytes prevent cerebral edema in chronically hyponatremic mice: rapid correction (experimental model of central pontine myelinolysis ) causes dehydration and shrinkage of brain. Metab. Brain Dis. 2:223-241.

18. Verbalis, J. G., and S. R. Gullans. 1991. Hyponatremia causes large 
sustained reductions in brain content of multiple organic osmolytes in rats. Brain Res. 567:274-282.

19. Lien, Y.-H. H., J. I. Shapiro, and L. Chan. 1990. Effects of hypernatremia on organic brain osmoles. J. Clin. Invest. 85:1427-1435.

20. Thurston, J. H., and R. E. Hauhart. 1987. Brain amino acids decrease in chronic hyponatremia and rapid correction causes brain dehydration: possible clinical significance. Life Sci. 40:2539-2542.

21. Kreis, R., N. A. Farrow, and B. D. Ross. 1990. Diagnosis of hepatic encephalopathy by proton magnetic resonance spectroscopy. Lancet. 336:635 636.

22. Michaelis, T., K.-D. Merboldt, W. Hänicke, M. L. Gyngell, H. Bruhn, and J. Frahm. 1991. On the identification of cerebral metabolites in localized ' $\mathrm{H}$ NMR spectra of human brain in vivo. NMR Biomed. 4:90-98.

23. Lee, J. H., E. Arcinue, and B. D. Ross. 1994. Organic osmolytes in the brain of an infant with hypernatremia. N. Eng. J. Med. 331:439-442.

24. Michaelis, T., J. Videen, M. S. Linsey, and B. D. Ross. 1994. Cerebral abnormalities in end stage renal disease. Proceedings, 2nd Society of Magnetic Resonance 1:306. (Abstr.)

25. Kreis, R., and B. D. Ross. 1992. Cerebral metabolic disturbances in patients with subacute and chronic diabetes mellitus by proton magnetic resonance spectroscopy. Radiology. 184:123-130.

26. Videen, J., T. Michaelis, A. S. Raj, P. Pinto, and B. D. Ross. 1994 Moderate hyponatremia modifies brain metabolism. Proceedings, 2nd Society of Magnetic Resonance. 1:193. (Abstr.)

27. Kreis, R., N. A. Farrow, and B. D. Ross. 1991. Localized 'H NMR spectroscopy in patients with chronic hepatic encephalopathy. Analysis of changes in cerebral glutamine, choline and inositols. NMR Biomed. 4:109-116.

28. Miller, B. L., R. Moats, T. Shonk, T. Ernst, S. Woolley, and B. D. Ross. 1993. Alzheimer disease: depiction of increased cerebral myo-inositol with proton MR spectroscopy. Radiology. 187:433-437.

29. Ernst, T., R. Kreis, and B. D. Ross. 1993. Absolute quantitation of water and metabolites in the human brain. Part I. Compartments and water. J. Magn Reson. 102:1-8.

30. Kreis, R., T. Ernst, and B. D. Ross. 1993. Absolute quantitation of wate and metabolites in the human brain. Part II. Metabolite concentrations. J. Magn Reson. 102:9-19.

31. Sterns, R. H., J. Baer, S. Ebersol, D. Thomas, J. W. Lohr, and D. E. Kamm 1993. Organic osmolytes in acute hyponatremia. Am. J. Physiol. 264:F833-F836.

32. Nitsch, R. M., J. K. Blusztajn, A. G. Pittas, B. E. Slack, J. H. Growdon, and R. J. Wurtman. 1992. Evidence for a membrane defect in Alzheimer disease brain. Proc. Natl. Acad. Sci. USA. 89:1671-1675.

33. Miller, B. L. 1991. A review of chemical issues in ${ }^{1} \mathrm{H}$ NMR spectroscopy: $N$-acetyl-L-aspartate, creatine and choline. NMR Biomed. 4:47-52.

34. Kreis, R., N. Farrow, and B. Ross. 1990. Diagnosis of hepatic encephalopathy by proton magnetic resonance spectroscopy. Lancet. 336:635-636.

35. Ross, B. D., F. Villamil, J. Korula, R. Kreis, T. Ernst, T. Shonk, and R. A. Moats. 1994. Proton MRS abnormalities in chronic and subclinical hepatic encephalopathy. Radiology. 193:457-463.

36. Adamson, D. J. A., R. B. S. Laing, and D. Nathwani. 1992. Alcoholism, hyponatremia and central neurological damage: more than pontine myelinolysis. Scott. Med. J. 37:83-84.

37. Tien, R., W. Kucharczyk, and J. Kucharczyk. 1992. Hyponatremic enceph alopathy: is central pontine myelinolysis a component? Am. J. Med. 92:513-522. 38. Narins, R. G. 1986. Therapy of hyponatremia, does haste make waste? $N$. Engl. J. Med. 314:1573-1574.

39. Van Reeth, O., and G. Decaux. 1989. Rapid correction of hyponatraemia with urea may protect against brain damage in rats. Clin. Sci. 77:351-355.

40. Soupart, A., A. Stenuit, O. Perier, and G. Decaux. 1991. Limits of brain tolerance to daily increments in serum sodium in chronically hyponatraemic rats treated with hypertonic saline or urea: advantages of urea. Clin. Sci. 80:77-84.

41. Oh, M. S., K. C. Choi, J. Uribarri, J. Sher, C. Rao, and H. J. Carroll 1990. Prevention of myelinolysis in rats by dexamethasone or colchicine. Am. J. Nephrol. 10:158-161. 